\title{
Strong words, but still a step back for researchers in emergency and critical care research? The proposed revision of the Declaration of Helsinki
}

Received: 23 May 2013

Accepted: 30 May 2013

Published online: 21 June 2013

(C) Springer-Verlag Berlin Heidelberg and ESICM 2013

E. J. O. Kompanje (『)

Department of Intensive Care, Erasmus MC University Medical Center, P.O. Box 2040, 3000, CA, Rotterdam, The Netherlands e-mail: e.j.o.kompanje@erasmusmc.nl

Tel.: +31-6-53837655

The World Medical Association (WMA) Declaration of Helsinki was first published in 1964 and set out rules and limits for medical research with humans. The declaration has been incorporated into many national laws and regulations concerning medical research with humans. It has, based on national divisions over the text, been changed eight times. Another revision is now on its way and the draft of the revised text is open for public consultation till 15 June 2013. The text is available on the WMA website (http://www.wma.net/en/20activities/10ethics/10helsinki/ 15publicconsult/DoH-draft-for-public-consultation_plain. pdf).

Several additions and revisions are worth mentioning in relation to critical care and emergency research, especially the report's approach to protection of patients in vulnerable populations.

New is that proven interventions must be evaluated in research ("even the best-proven interventions must be evaluated continually through research for their safety, effectiveness, efficiency, accessibility, and quality'). The word 'current' is exchanged for 'proven'. This change means that accepted guidelines and protocols, even when they are based on proven facts and practice, should be evaluated on a regular basis. However, it is not clear why this obligation is added and how this can be practically carried out.

The wording of the text is strengthened by changing the word 'should' to 'must' ('physicians must consider the ethical, legal and regulatory norms and standards for research involving human subjects in their own countries as well as applicable international norms and standards') aiming at international ethical collaboration.

The necessary qualifications of the researcher are expanded with addition of the word 'education' ('medical research involving human subjects must be conducted only by individuals with the appropriate scientific education, training and qualifications'). Training (e.g. training on the job) is not enough anymore; there must be proof of education. Obviously, these qualifications must be obtained prior to the study.

Paragraph 15 concerns financial compensation for harmed research subjects. As it is often difficult to separate complications and evolving symptoms of serious illness from adverse events resulting from the experimental treatment of intervention, this may result in more negative external attention (e.g. law suits). Paragraph 17 focusses on risk management and responsibility ('measures to minimise the risks must be implemented. The risks must always be monitored by the researcher throughout the trial'). It is obviously the responsibility of the researcher to develop a data and safety monitoring plan, not only covered by the sponsor and a monitoring board, but also by the researcher himself throughout the trial, monitoring the progress and safety. In the European Union trial Directive 2001/20/EC, the responsibility to monitor, record and report adverse events is more the responsibility of the sponsor than the researcher, which makes paragraph 17 of the declaration somewhat divergent.

Paragraphs 19-20 concern vulnerable populations. The words 'need special protection' in the old text are 
exchanged for 'have an increased likelihood of incurring additional and greater harm'. The sentence 'all vulnerable groups need specifically considered protection' is added. Although specific groups are not mentioned, patients with critical conditions are certainly addressed in this paragraph.

Fair and reasonable benefit are now combined: 'Medical research involving a disadvantaged or vulnerable population or community is only justified if the research is responsive to the health needs and priorities of this population or community and the research cannot be carried out in a non-vulnerable population. In addition this population or community should stand to benefit from the knowledge, practices or interventions that result from the research'. ICU patients are a vulnerable population with no representatives in non-vulnerable populations. Although we can argue that observational research benefits patients by allowing us to identify problems and potential solutions, research ethics committees (REC) are sometimes reluctant if this advantage is not obvious for individual patients. The revised text of the declaration, which is in line with the revised draft text of Directive 2001/20/EC, gives the researcher and sponsor the possibility to avoid the obligation of providing a direct benefit of research. It can help RECs to authorize emergency research with no direct benefit to individual patients.

The design and performance of a research study must be clearly described in the research protocol. Added is that 'the research protocol should discuss and justify the chosen study design', making pure observational studies without direct benefit difficult to conduct. The researchers must, at the end of the study, submit a final report to the REC concerning the study findings and conclusions. This is a good thing. However, these RECs can be more resistant in permitting critical care and emergency research when reasonable and fair benefit is not clearly described. And, what the REC should do with a provided report is not clear. Must they analyse and interpret the results? Also, is it not clear what the REC should do when the results are not in line with the protocol, are unethical or if the results do not provide any benefit for the study population.

Research using identifiable human material, such as biomarkers and genetics, is only possible after informed consent of the subject. However, an exception is made for situations where consent would be impossible or impracticable to obtain. In such situations the research may be done after consideration and approval of a REC, but without consent of the patient or its representative. This permissiveness is surprising in the light of the other revisions in the revised declaration where strict terms replace more superficial wording. Researchers can think that consent is not needed, as it is often impracticable or impossible to obtain consent of the patient. Furthermore, the space given by the declaration is not in line with allowing the processing of personal data concerning health in the European Union Data Protection Directive 95/46/EC (section III, article 8).

The use of an unproven intervention in a patient is still possible in the revised declaration. Only the word 'individual' is added to 'patient', making it more explicit to a particular situation. Unproven intervention should be made, when possible, the object of research. In the new text 'when possible' is deleted and 'subsequently' is added, making it inescapable to neglect the obligation to evaluate the unproven intervention. This is a good thing, but is also of concern as 'unproven' interventions often are applied in life-threatening situations. Should every 'off-label' use of medication and every 'unproven' attempt to save a life be subjected to subsequent research?

In conclusion, the revisions reflect ongoing concerns over clinical research involving vulnerable patients. The wording is stronger and more direct than in previous versions. Of course this is needed in times when violations of patients' rights and scientific fraud are becoming less scarce than years ago. However, clinical research in the field of emergency medicine has always been difficult and will become more so. We all have encountered the constraints in relation to the European Directive 2001/20/ EC. The revision of the Helsinki declaration in combination with the new proposal for a regulation of the European Parliament and of the council on clinical trials on medicinal products for human use, and repealing Directive 2001/20/EC (http://ec.europa.eu/health/files/ clinicaltrials/2012_07/proposal/2012_07_proposal_en.pdf), will mean that the RECs will probably have more difficulties in evaluating emergency research in acute lifethreatening situations in patients lacking the ability to consent. Even ethically correct research proposals shall encounter delay and rejection. Again, emergency research will be hindered.

Conflicts of interest The author declared no conflicts of interest. 